\title{
The OmegaWhite Survey for short-period variable stars - IV. Discovery of the warm DQ white dwarf OW J175358.85-310728.9
}

\author{
S. A. Macfarlane, ${ }^{1,2}$ P. A. Woudt, ${ }^{2 \star}$ P. Dufour, ${ }^{3}$ G. Ramsay, ${ }^{4}$ P. J. Groot, ${ }^{1}$ \\ R. Toma,${ }^{4}$ B. Warner, ${ }^{2}$ K. Paterson, ${ }^{2}$ T. Kupfer, ${ }^{5}$ J. van Roestel, ${ }^{1}$ \\ L. Berdnikov, ${ }^{6,7,8}$ T. Dagne ${ }^{6}$ and F. Hardy ${ }^{3}$ \\ ${ }^{1}$ Department of Astrophysics/IMAPP, Radboud University, PO Box 9010, NL-6500 GL Nijmegen, the Netherlands \\ ${ }^{2}$ Department of Astronomy, University of Cape Town, Private Bag X3, Rondebosch 7701, South Africa \\ ${ }^{3}$ Departement de Physique, Universite de Montreal, C.P. 6128, Succ. Centre-Ville, Montreal, QC H3C 3J7, Canada \\ ${ }^{4}$ Armagh Observatory, College Hill, Armagh BT61 9DG, Northern Ireland, UK \\ ${ }^{5}$ Division of Physics, Mathematics, and Astronomy California Institute of Technology, Pasadena, CA 91125, USA \\ ${ }^{6}$ Astronomy and Astrophysics Research division, Entoto Observatory and Research Center, PO Box 8412 Addis Ababa, Ethiopia \\ ${ }^{7}$ Sternberg Astronomical Institute, Lomonosov Moscow State University, Universitetskij Pr. 13, Moscow 119992, Russia \\ ${ }^{8}$ Isaac Newton Institute of Chile, Moscow Branch, Universitetskij Pr. 13, Moscow 119992, Russia
}

Accepted 2017 March 23. Received 2017 March 18; in original form 2016 September 16

\begin{abstract}
We present the discovery and follow-up observations of the second known variable warm DQ white dwarf OW J175358.85-310728.9 (OW J1753-3107). OW J1753-3107 is the brightest of any of the currently known warm or hot DQ and was discovered in the OmegaWhite Survey as exhibiting optical variations on a period of 35.5452 (2) $\mathrm{min}$, with no evidence for other periods in its light curves. This period has remained constant over the last $2 \mathrm{yr}$ and a singleperiod sinusoidal model provides a good fit for all follow-up light curves. The spectrum consists of a very blue continuum with strong absorption lines of neutral and ionized carbon, a broad $\mathrm{He}_{\mathrm{I}} \lambda 4471$ line and possibly weaker hydrogen lines. The $\mathrm{C}_{\mathrm{I}}$ lines are Zeeman split, and indicate the presence of a strong magnetic field. Using spectral Paschen-Back model descriptions, we determine that OW J1753-3107 exhibits the following physical parameters: $T_{\text {eff }}=15430 \mathrm{~K}$, $\log (g)=9.0, \log (N(\mathrm{C}) / N(\mathrm{He}))=-1.2$ and the mean magnetic field strength is $B_{z}=2.1 \mathrm{MG}$. This relatively low temperature and carbon abundance (compared to the expected properties of hot DQs) is similar to that seen in the other warm DQ SDSS J1036+6522. Although OW J1753-3107 appears to be a twin of SDSS J1036+6522, it exhibits a modulation on a period slightly longer than the dominant period in SDSS J1036+6522 and has a higher carbon abundance. The source of variations is uncertain, but they are believed to originate from the rotation of the magnetic white dwarf.
\end{abstract}

Key words: stars: carbon - stars: evolution - stars: individual: OW J175358.85-310728.9 stars: magnetic field - stars: rotation - white dwarfs.

\section{INTRODUCTION}

White dwarfs (WDs) have been shown to exhibit short-period variations since the mid-20th century, when the first variable WD, HL Tau 76, was observed to pulsate on a period of $12.5 \mathrm{~min}$ (Landolt 1968). HL Tau 76 is now known as a WD variable containing a hydrogenenriched atmosphere (DAV, or ZZ Ceti stars) and has since been observed to pulsate in multiple modes (e.g. Dolez et al. 2006). Other variable types of WD stars include helium-dominated WDs

^E-mail: pwoudt@ast.uct.ac.za
(DBV or V777 Her), WDs with atmospheres that are rich in carbon, helium and oxygen (GW Vir stars, see Fontaine \& Brassard 2008; Winget \& Kepler 2008; Althaus et al. 2010, for detailed reviews of pulsating WDs), extremely low-mass WDs (Hermes et al. 2012) and a new class of WDs that exhibit carbon-enriched atmospheres ("hot DQ, Montgomery et al. 2008 or 'warm DQ', Williams et al. 2013).

Although the variations exhibited in DAV and DBV stars can be attributed to non-radial gravity wave pulsations, it has recently been shown that the origin of variations in warm and hot carbonenriched DQs are likely due to the rotation of the WD (e.g. Williams et al. 2016). As only eight of these warm and hot DQ WDs have so far been observed to show periodic variations, it is important that 
more objects are found and studied. Such research can then be used to test the proposed evolutionary channels of (DQ) WDs.

The typical cool DQ WD exhibits neutral carbon or molecular $\mathrm{C}_{2}$ (Swan bands) in its spectra, and a temperature below $\sim 13000 \mathrm{~K}$ (see e.g. Dufour, Bergeron \& Fontaine 2005). However, Liebert et al. (2003) discovered a new class of DQ in the Sloan Digital Sky Survey (SDSS; Alam et al. 2015) that exhibit neutral and/or ionized atomic carbon lines in their spectra. Using this sample, as well as another similarly hot DQs discovered by Williams et al. (2006), Dufour et al. (2007, 2008) found that these so-called hot DQ WDs have carbon-dominated atmospheres, a carbon abundance $\log (N(\mathrm{C}) / N(\mathrm{He})) \geq 1$, and all lie within a specific temperature range $\left(18000 \mathrm{~K}<T_{\text {eff }}<24000 \mathrm{~K}\right)$. Furthermore, $\sim 70$ per cent of these hot DQs have been found to be strongly magnetic with magnetic field strengths $\geq 1 \mathrm{MG}$ (Dufour et al. 2011; Williams et al. 2016).

Following the discovery of the first six hot DQs (Barlow et al. 2008; Montgomery et al. 2008; Dunlap, Barlow \& Clemens 2010; Dufour et al. 2011; Lawrie et al. 2013), a seventh DQ, namely SDSSJ103655.39+652252.2 (hereafter SDSS J1036+6522; Williams et al. 2013), was discovered at a cooler temperature and with a lower carbon abundance than that of the previously discovered hot DQs $\left(T_{\text {eff }} \approx 15500 \mathrm{~K}\right.$, $\log (N(\mathrm{C}) / N(\mathrm{He}))=-1.0)$. Thus, it is believed to be a 'warm $\mathrm{DQ}$, in a transition state from the hot DQs to a set of cooler helium-dominated DQ WDs with a temperature in the range $13000 \mathrm{~K}<T_{\text {eff }}<18000 \mathrm{~K}$ (Dufour et al. 2013; Williams et al. 2013; Fortier \& Dufour 2015).

In this paper, we present the discovery and initial follow-up observations of a second variable warm DQ, OW J175358.85-310728.9 (hereafter OW J1753-3107) discovered in the OmegaWhite (OW) Survey (Macfarlane et al. 2015). As this is the second warm or hot DQ to be discovered outside the SDSS survey (see also Williams et al. 2006), in Section 2, we discuss details of the discovery and describe the survey in which it was found. We present photometric follow-up observations in Section 3, where we analyse the light curves for periods and compare pulse shape properties spanning $2 \mathrm{yr}$ of observations. In Section 4, spectral lines are identified in a high-resolution spectrum of OW J1753-3107, and results from the modelling of spectral lines are presented. Lastly, we discuss the (magnetic) nature of OW J1753-3107, as well as the origin of variations.

\section{DISCOVERY OF DQ OW J1753-3107}

The OW Survey (Macfarlane et al. 2015; Toma et al. 2016) is a wide-field high-cadence survey that aims to cover $400 \mathrm{deg}^{2}$ of the Galactic Plane with the $g$-band observations, reaching a depth of 21.5 mag. OW sources are cross-matched with the VST Photometric $\mathrm{H} \alpha$ Survey of the Southern Galactic Plane (VPHAS+; Drew et al. 2014) in order to obtain multiband colour information. Furthermore, all instrumental magnitudes in OW are calibrated to standard Vega magnitudes using the AAVSO All-Sky Survey (Henden et al. 2012). The aim of the OW survey is to identify short-period variable systems, varying on time-scales of minutes to less than an hour, such as ultracompact binary (UCB) star systems and rare pulsating WD/subdwarf sources. Each square degree field is observed with 39-s exposures over a 2-h duration (with a mean cadence of 3.6 $\pm 0.7 \mathrm{~min}$; Toma et al. 2016) using OmegaCAM on the VLT Survey Telescope (VST; Kuijken et al. 2002; Capaccioli \& Schipani 2011)

OW J1753-3107 was initially selected for follow-up observations as it exhibited very blue colours $(g-r=-0.16$, $u-g=-1.33)$ and short-period variations $\left(P_{\mathrm{OW}} \approx 35 \mathrm{~min}\right)$
Table 1. Target properties for OW J1753-3107: star ID; RA and Dec.; OW Lomb-Scargle $(\mathrm{LS})$ period $\left(P_{\mathrm{OW}}\right)$, OW-calibrated $g$-band magnitude $\left(\mathrm{OW}_{g}\right)$, amplitude of OW light curve $\left(A_{\mathrm{OW}}\right)$, VPHAS + colour indices $g-r$ and $u-g$. All instrumental magnitudes in OW are calibrated using the AAVSO All-Sky Survey (Henden et al. 2012) to the standard (Vega) scale.

\begin{tabular}{lc}
\hline Star ID & OW J175358.85-310728.9 \\
\hline RA (J2000) & $17^{\mathrm{h}} 53^{\mathrm{m}} 58^{\mathrm{s}} .85$ \\
Dec. (J2000) & $-31^{\circ} 07^{\prime} 28^{\prime \prime} .9$ \\
OW $_{g}$ & $15.8 \mathrm{mag}$ \\
$P_{\mathrm{OW}}$ & $35.2 \mathrm{~min}$ \\
$A_{\mathrm{OW}}$ & 2.6 per cent \\
VPHAS+ Colours, on standard (Vega) scale & \\
$u$ & $14.399 \pm 0.003$ \\
$g$ & $15.735 \pm 0.005$ \\
$r^{a}$ & $15.890 \pm 0.008$ \\
$i$ & $15.796 \pm 0.008$ \\
$g-r$ & $-0.155 \pm 0.013$ \\
$u-g$ & $-1.336 \pm 0.008$
\end{tabular}

Note. ${ }^{a}$ Mean $r$-band colour obtained from two separate VPHAS + epochs (Drew et al. 2014).

typically associated with AM CVn systems (see Table 1 for target properties). In Fig. 1, we show the OW light curve, along with a finding chart created using a subframe of the OW $g$-band reference frame containing OW J1753-3107.

Using the Spectrograph Upgrade - Newly Improved Cassegrain spectrograph (SpUpNIC; Crause et al., in preparation) on the South African Astronomical Observatory (SAAO) 1.9-m telescope on 2016 April 30, we obtained two 1800-s long-slit exposures using a slit width of 1.5 arcsec with grating 7 for a dispersion of $2.8 \AA$ pixel $^{-1}$ across the wavelength range $(\sim 3500-8000 \AA)$. The median-combined, flux-calibrated spectrum, shown in Fig. 1, reveals a blue continuum, along with evidence for absorption lines of $\mathrm{C}_{\mathrm{I}}, \mathrm{C}_{\mathrm{II}}, \mathrm{He}_{\mathrm{I}} \lambda 4471$, and possibly $\mathrm{H} \alpha$. Furthermore, the presence of Zeeman-split lines is evidence that OW J1753-3107 contains a relatively strong magnetic field. Further photometric and spectroscopic observations of OW J1753-3107 were carried out in order to precisely characterize the nature of this DQ WD source.

\section{PHOTOMETRIC ANALYSIS}

Seven high-cadence photometric observing runs of OW J1753-3107 were conducted over 2 yr: during 2015 June, 2016 May, 2016 June and 2016 July using the Sutherland High-speed Optical Cameras (SHOC; Coppejans et al. 2013) on the 1.0-m and 1.9-m SAAO telescopes, located at the Sutherland Observatory in South Africa. The observing details of these photometric runs are listed in Table 2, and hereafter will be named LC_OW (for the original OW observation), LC_SHOC_1, LC_SHOC_2, LC_SHOC_3, LC_SHOC_4, LC_SHOC_5, LC_SHOC_6 and LC_SHOC_7 (in order of observation date, as indicated in Table 2). All SHOC observations were obtained using a $1 \mathrm{MHz}$ read-out speed and a pre-amplifier gain of 2.4 (see Coppejans et al. 2013, for further details about the SHOC cameras).

The seven follow-up SHOC light curves are shown in Fig. 2, either in 1-min bins (LC_SHOC_1, LC_SHOC_2), or in 2-min bins (LC_SHOC_3 - LC_SHOC_7). The raw data were reduced and light curves extracted using the standard IRAF routines (Tody 1986, 1993), including bias-subtraction, flat-fielding and aperture photometry. Using the technique of differential photometry, the average light curve of three bright (stable) neighbouring stars was subtracted 

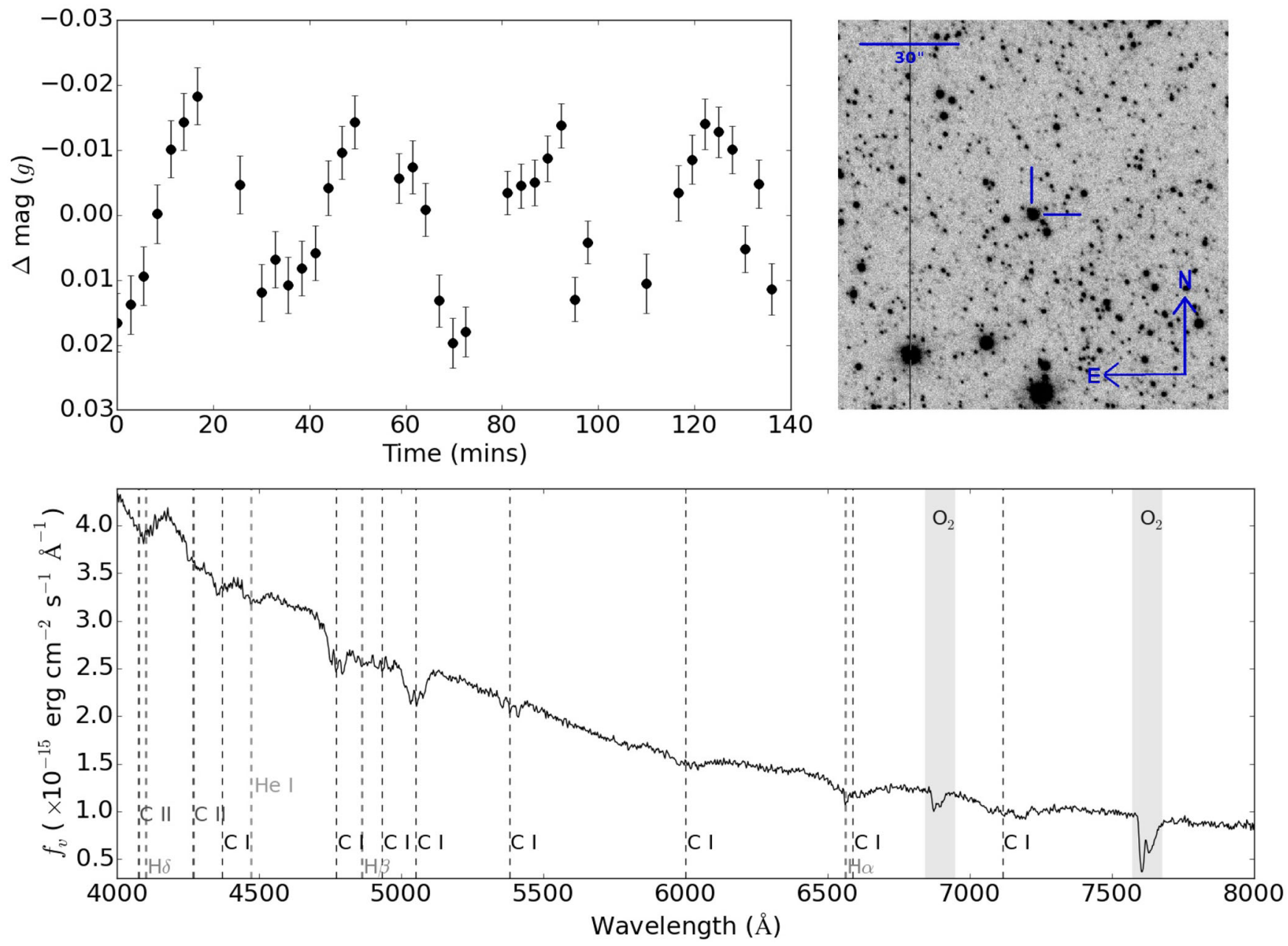

Figure 1. The $g$-band OW light curve (upper left panel), finding chart (upper right panel) and SpUpNIC-observed spectrum (lower panel) of the warm DQ OW J1753-3107. The finding chart is a $2 \operatorname{arcmin} \times 2$ arcmin subframe of an OW reference frame, observed in the $g$ band. The spectrum has been flux and wavelength calibrated. Dashed lines indicate important spectral lines (as labelled), and shaded areas cover atmospheric telluric bands.

Table 2. Photometric observing log with parameters: run identification name (Run Id), date of observation (Date obs.), time at the start of the observation $\left(\mathrm{HJD}_{0}\right)$, the telescope (Tel.), instrument (Instr.) and filter used, the number of pixels binned in each image (binning), the length of each exposure (Exp. time) and the duration of the observing run (duration).

\begin{tabular}{|c|c|c|c|c|c|c|c|c|}
\hline Run ID & $\begin{array}{c}\text { Date obs. } \\
\text { (dd/mm/yyyy) }\end{array}$ & $\mathrm{HJD}_{0}$ & Tel. & Instr. & Filter & Binning & $\begin{array}{l}\text { Exp. time } \\
\text { (s) }\end{array}$ & $\begin{array}{l}\text { Duration } \\
\text { (h) }\end{array}$ \\
\hline LC_OW & 29/07/2014 & 2456867.9920 & VST & OmegaCAM & $g^{a}$ & $1 \times 1$ & $39^{b}$ & 2.3 \\
\hline LC_SHOC_2 & $16 / 05 / 2016$ & 2457525.4137 & SAAO $1.0-\mathrm{m}$ & SHOC & White light & $1 \times 1$ & 10,15 & 6.4 \\
\hline LC_SHOC_3 & 08/06/2016 & 2457548.3247 & SAAO $1.0-\mathrm{m}$ & SHOC & B & $2 \times 2$ & 30 & 7.9 \\
\hline LC_SHOC_4 & $12 / 06 / 2016$ & 2457552.3227 & SAAO $1.0-\mathrm{m}$ & SHOC & $B$ & $2 \times 2$ & 60,30 & 6.8 \\
\hline LC_SHOC_5 & $13 / 07 / 2016$ & 2457583.4301 & SAAO $1.0-\mathrm{m}$ & SHOC & $B$ & $2 \times 2$ & 30 & 4.2 \\
\hline LC_SHOC_7 & $16 / 07 / 2016$ & 2457586.3603 & SAAO $1.0-\mathrm{m}$ & SHOC & $B$ & $2 \times 2$ & 45 & 4.8 \\
\hline
\end{tabular}

Notes. ${ }^{a} \mathrm{OW}$ magnitude in standard (Vega) system; ${ }^{b}$ With a cadence of $\sim 167 \mathrm{~s}$.

from the target light curve in order to compensate for atmospheric effects during the observing runs. Although LC_SHOC_1 and LC_SHOC_2 were observed without a filter, the most recent runs, LC_SHOC_3 to LC_SHOC_7, were observed using a blue filter (Bessell $B$ ). Since OW J1753-3107 is a bluer object than the majority of its neighbouring stars, it can be easily identified in $B$ filter fields. Furthermore, the runs using a $B$ filter were relatively unaffected by the changing airmass throughout the night, unlike the runs observed without a filter which were heavily affected (airmass trends have been removed from the light curves shown in Fig. 2). This is due to second-order colour terms between the target and the (redder) comparison stars.

From visual inspection of the light curves in Fig. 2, the original variations identified in the OW light curve of OW J1753-3107, fluctuating on a period of $\sim 35 \mathrm{~min}$, can clearly be seen on all follow-up SHOC light curves. In the case of LC_SHOC_2, the 


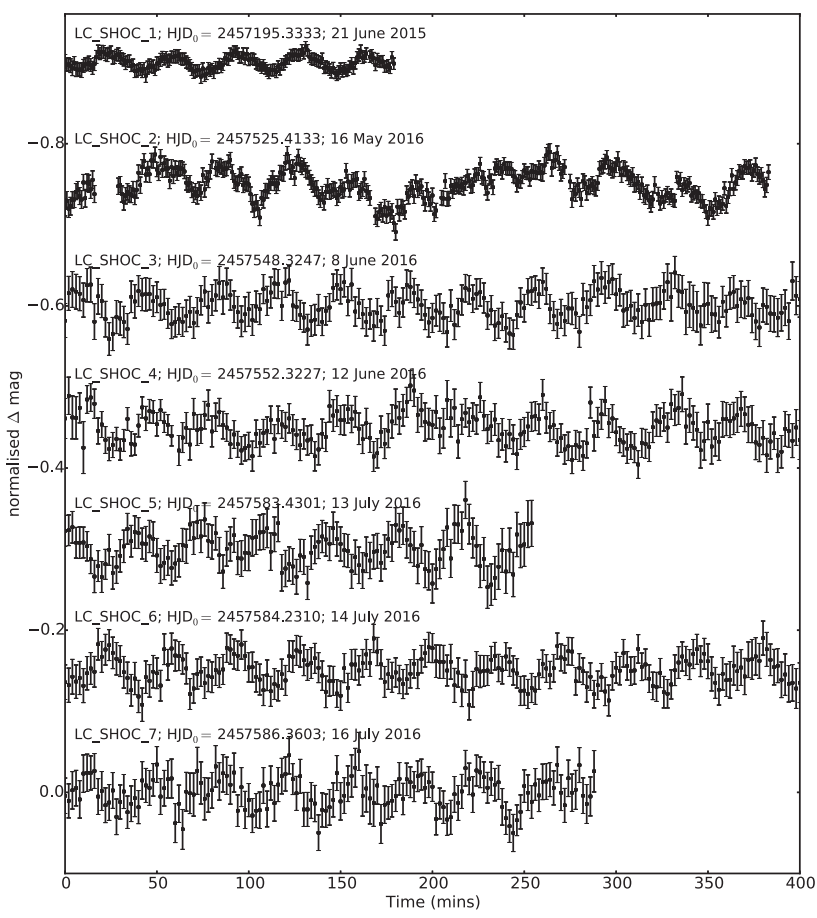

Figure 2. All follow-up light curves obtained for OW J1753-3107, using SHOC cameras on either the SAAO 1.0-m or 1.9-m telescope. Data points in the light curves are either in 1-min bins (LC_SHOC_1 and LC_SHOC_2) or in 2-min bins (LC_SHOC_3-LC_SHOC_7) and large trends due to changing airmasses have been removed. Light curves have been shifted in the $y$-axis for visualization purposes, and are plotted from top to bottom according to their chronological date of observation as labelled $\left(\mathrm{HJD}_{0}\right.$ is the time at the start of each observation). LC_SHOC_1 and LC_SHOC_2 were observed with no filter, and LC_SHOC_3 through to LC_SHOC_7 were observed using a Bessel $B$ filter. See Table 2 for the observation log.

Table 3. Peak LS periodogram period $\left(P_{\mathrm{LS}}\right)$ with error, and sinusoidal fitting parameters ${ }^{a}$ for all photometric runs on OW J1753-3107, including the peak frequency in the power spectrum $(f)$, the amplitude of the peak frequency in the power spectrum $(A)$ and the phase of the fitted light curve $(\psi)$. Also shown is the median residual of the model fit to the folded lightcurve data (resid $\left.{ }_{\text {median }}\right)$, shown in Fig. 3.

\begin{tabular}{lcccrc}
\hline RunID & $\begin{array}{c}P_{\text {LS }} \\
(\mathrm{min})\end{array}$ & $\begin{array}{c}f \\
\left(\text { cycles d }^{-1}\right)\end{array}$ & $\begin{array}{c}A \\
(\text { per cent })\end{array}$ & $\begin{array}{c}\psi \\
(\mathrm{rad})\end{array}$ & $\begin{array}{r}\mathrm{resid}_{\text {median }} \\
(\text { per cent })\end{array}$ \\
\hline LC_OW & $35.3 \pm 3.9$ & 0.0245 & 1.3 & 0.09 & 0.5 \\
LC_SHOC_1 & $35.3 \pm 3.3$ & 0.0245 & 1.0 & -1.18 & 0.2 \\
LC_SHOC_2 & $35.5 \pm 1.4$ & 0.0247 & 1.4 & 0.23 & 0.9 \\
LC_SHOC_3 & $35.6 \pm 1.3$ & 0.0247 & 1.9 & 1.34 & 1.6 \\
LC_SHOC_4 & $35.7 \pm 1.4$ & 0.0248 & 1.9 & 1.30 & 1.0 \\
LC_SHOC_5 & $35.6 \pm 2.1$ & 0.0247 & 2.2 & 2.55 & 0.7 \\
LC_SHOC_6 & $35.6 \pm 1.2$ & 0.0247 & 2.1 & 2.13 & 0.7 \\
LC_SHOC_7 & $35.5 \pm 2.0$ & 0.0247 & 1.9 & 0.61 & 1.0 \\
\hline
\end{tabular}

Note. ${ }^{a}$ Sinusoidal model fit, see Section 3 for model description.

observing conditions on the night of 2016 May 16 were poor, which ultimately injected a false long-period variation of $\sim 174$ min into the light curve (as shown in Fig. 2). The non-validity of this period is further confirmed as we do not observe it in the other longduration SHOC light curves. The amplitude of the light curves in LC_SHOC_3 through to LC_SHOC_7 appear to be slightly larger than that of the previous runs, but this is to be expected as the observations were made with two different filters (see Table 3). In order to obtain better estimates on periods present in the light curves,

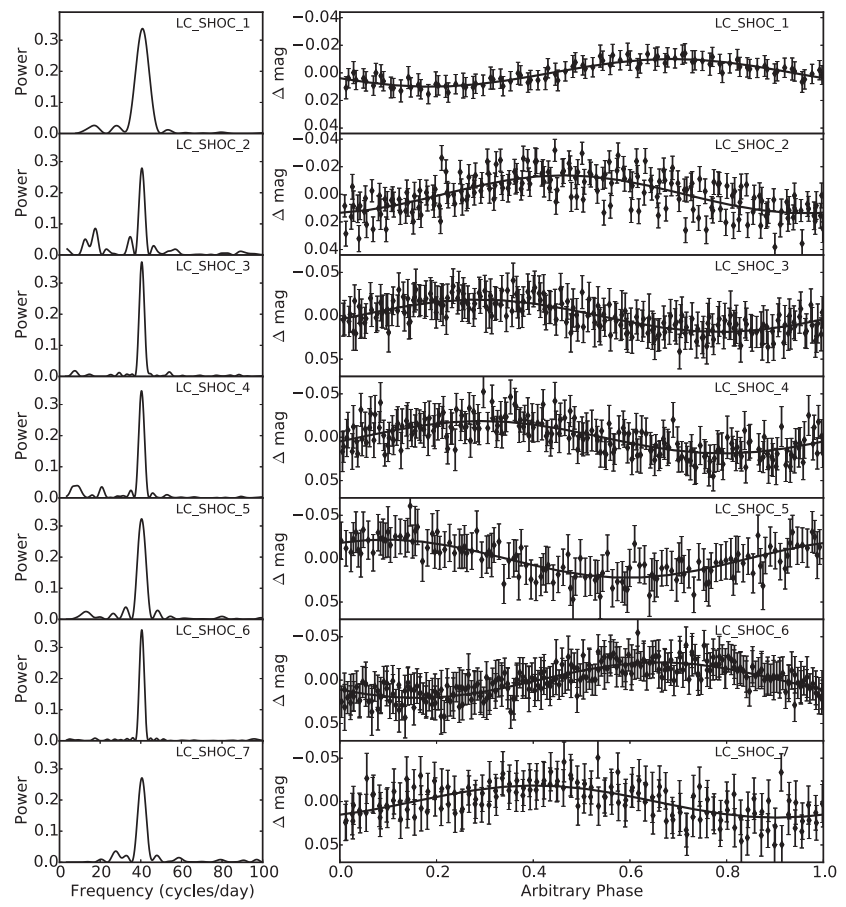

Figure 3. LS power spectrum (left-hand panels) and folded light curves (right-hand panels) of the seven follow-up SHOC runs on OW J1753-3107. In all panels, observing runs are labelled as indicated in Table 2. The power spectrum shows the non-normalized LS periodogram power on the $y$-axis, and frequency in cycles $\mathrm{d}^{-1}$ on the $x$-axis. The light curves (right-hand panels) are folded on the period of the peak power shown in their respective spectrum (and in Table 3), and are in 2-min bins. A single-period sinusoidal model is fit to each of the folded light curves. The data have been observed with either no filter (LC_SHOC_1, LC_SHOC_2) or in B (LC_SHOC_3 LC_SHOC_7).

we obtained photometric variability parameters using the VARTOOLS package (Hartman et al. 2008) on the follow-up observations. These parameters include the period corresponding to the peak LS power spectrum $\left(P_{\mathrm{LS}}\right)$.

A period of $\sim 35$ min corresponding to the peak power is confirmed in the LS power spectrum of each SHOC light curve, as shown in Fig. 3 (after the spurious 174-min period is removed from LC_SHOC_2). The LS periods are listed, with errors, in Table 3. We do not find any significant peaks above 100 cycles $\mathrm{d}^{-1}$ and, although there are a few other smaller peaks in the power spectrum, they are not featured in every spectrum as is the case of the overwhelming peak period. Thus, we conclude that OW J1753-3107 has a single photometric period, equal to $\sim 35 \mathrm{~min}$. Furthermore, this period has remained approximately constant from the first OW observation in 2014 July, to 2 yr later with the LC_SHOC_7 run in 2016 July.

In order to test the stability and accuracy of the single-period assumption, we folded each light curve on their respective $P_{\mathrm{LS}}$, as shown in Fig. 3 in 2-min bins for visualization purposes. We fit each folded light curve with a single-mode sinusoidal curve using parameters determined through VARTOOLS, and a simple sinusoidal model of the form:

$f(t)=A \cos (\omega t+\psi)$,

where $f(t)$ is the model light curve flux as a function of time $t, \omega$ is the frequency associated with the peak LS periodogram power, $A$ is the amplitude of the model light curve and $\psi$ gives the phase of the rotation at the moment of observation. 

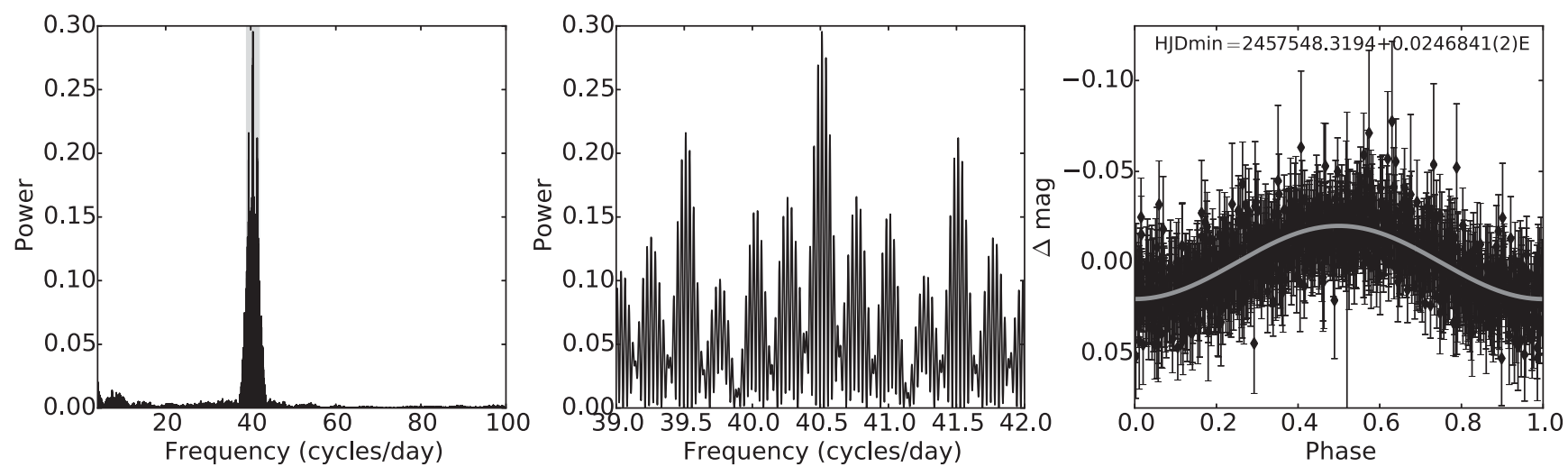

Figure 4. LS power spectra (left-hand and middle panels) and phase-folded light curve (right-hand panel) of the combined B-filter SHOC runs on OW J17533107 (LC_SHOC_3-LC_SHOC_7). The power spectra shows the non-normalized LS periodogram power on the $y$-axis, and frequency in cycles $\mathrm{d}^{-1}$ on the $x$-axis. The spectrum in the middle panel is a zoomed-in section of the spectrum in the left-hand panel (grey shading). The light curve (right-hand panel, in 2 -min bins) has been folded on the ephemeris and period indicated in the figure $\left(\mathrm{HJD}_{\min }\right)$, which corresponds to the period of the peak power shown in the spectrum. A single-period sinusoidal model is fit to the folded light curve (grey line).

Table 4. Spectroscopic observing log, including the following parameters: date of observation (Date obs.), time at the start of the observation $\left(\mathrm{HJD}_{0}\right)$, the telescope (Tel.), instrument (Instr.) and mode used, the grating (Gr.) and grating angle (Gr. angle) used, the slit width, the length of each exposure (Exp. time) and the dispersion across the wavelength range (Disp.).

\begin{tabular}{lccccccccc}
\hline $\begin{array}{l}\text { Date } \\
\text { obs. } \\
(\mathrm{dd} / \mathrm{mm} / \mathrm{yyyy})\end{array}$ & $\mathrm{HJD}_{0}$ & Tel. & Instr. & Mode & Gr. & $\begin{array}{c}\text { Gr. } \\
\text { angle } \\
\left({ }^{\circ}\right)\end{array}$ & $\begin{array}{c}\text { Slit } \\
\text { width } \\
(\operatorname{arcsec})\end{array}$ & $\begin{array}{c}\text { Exp. } \\
\text { time } \\
(\mathrm{s})\end{array}$ & $\begin{array}{c}\text { Disp. } \\
(\AA \mathrm{A} \text { pixel }\end{array}$ \\
\hline $30 / 04 / 2016$ & 2457509.4982 & SAAO 1.9-m & SpUpNIC & long slit & gr7 & 17.4 & 1.8 & $2 \times 1800$ & 2.80 \\
$15 / 05 / 2016$ & 2457540.4526 & SALT & RSS & long slit & pg1300 & 18.88 & 1.5 & $3 \times 720$ & 0.66 \\
$01 / 06 / 2016$ & 2457540.9525 & Keck II & ESI & Echellete & & & 1 & $7 \times 240,12 \times 180$ & 0.16 \\
\hline
\end{tabular}

The fitting parameters for the light curve of each observing run (including LC_OW) are listed in Table 3. In all SHOC light curves, the folded data agree well with a single-mode sinusoidal model fit, having median residual values between 0.2 per cent and 1.6 per cent (see Table 3). The unexpected scatter seen in LC_SHOC_2 is due to poor atmospheric conditions during the observing run. The ephemeris is found to be $\mathrm{HJD}_{\min }=2457548.31942+0.0246841(2)$ E. Furthermore, by combining the five $B$ filter runs (LC_SHOC_3LC_SHOC_7), we find OW J1753-3107 exhibits a single-mode frequency of $4.68887(3) \times 10^{-4} \mathrm{~Hz}$, corresponding to 35.5452 (2) min. This is further shown in the folded light curve and power spectrum of the combined $B$ filter runs in Fig. 4.

\section{SPECTROSCOPIC ANALYSIS}

The spectrum of OW J1753-3107, as shown in Fig. 1 and discussed in Section 2, reveals clear evidence for carbon and helium, which is very similar in appearance to the spectrum exhibited by the first warm DQ SDSS J1036+6522 (Williams et al. 2013). Hence, we are able to fit the spectral features exhibited by OW J1753-3107 using an adaptation of the model that was applied to SDSS J1036+6522 by Williams et al. (2013). Fitting the spectral features allows us to obtain better estimates of the physical properties of OW J1753-3107, including the temperature $\left(T_{\text {eff }}\right)$, surface gravity $(g)$, magnetic field strength $\left(B_{z}\right)$ and carbon abundance $(N(\mathrm{C}) / N(\mathrm{He}))$. Furthermore, we obtained high-resolution spectra for precise spectral line identification purposes. Details of the three spectroscopic follow-up observing runs are shown in Table 4 , and are discussed in detail in Sections 4.1 and 4.2.

\subsection{Spectral line identification}

In order to precisely identify any absorption lines shown in the spectrum of OW J1753-3107 and to search for radial velocity shifts, we obtained 18 3-min or 4-min spectra using the Echellette Spectrograph and Imager instrument (ESI; Sheinis et al. 2002) located on the Keck II telescope. Exposures were taken in Echellette mode, with a slit width of 1 arcsec, and the spectra were reduced and extracted using the MAKEE ${ }^{1}$ pipeline. A wavelength solution was found for the spectra by calibrating with $\mathrm{HgNeXeCuAr}$ arcs, and instrumental flexure was corrected for using $\mathrm{CuAr}$ arcs observed at the beginning of the night.

The average-combined and the continuum-normalized spectrum are shown in Fig. 5, with prominent absorption lines identified and labelled; the absorption line at $\sim 6105 \AA$ remains unidentified. The spectrum is the combination of the 10 orders on the CCD, and span the wavelength range from 4170 to $7200 \AA$. The combination of the orders can cause relatively higher noise levels where they have been stitched together, as shown in the region around $\sim 4500 \AA$. In the spectrum, we are able to make out clear Zeeman-split lines of $\mathrm{C}_{\mathrm{I}}$ and $\mathrm{C}_{\text {II. The }}$ There is also possible evidence for the $\mathrm{H} \alpha$ and $\mathrm{H} \beta$ lines identified in the SpUpNIC spectrum (Fig. 1). However, there is no apparent evidence for the $\mathrm{He}$ I $\lambda 4471$ broad absorption dip that was present in the SpUpNIC spectrum. This is likely a consequence of the continuum fit to the individual orders.

In the $18 \mathrm{KECK}$ ESI spectra, radial velocities were measured by fitting a Lorentzian/Gaussian function to the spectral lines of $\mathrm{H} \alpha$, $\mathrm{H} \beta$ and $\mathrm{C}_{\mathrm{I}} \lambda 5380$ using FITSB 2 (Napiwotzki et al. 2004). These

\footnotetext{
${ }^{1}$ http://www.astro.caltech.edu/ tb/makee/
} 

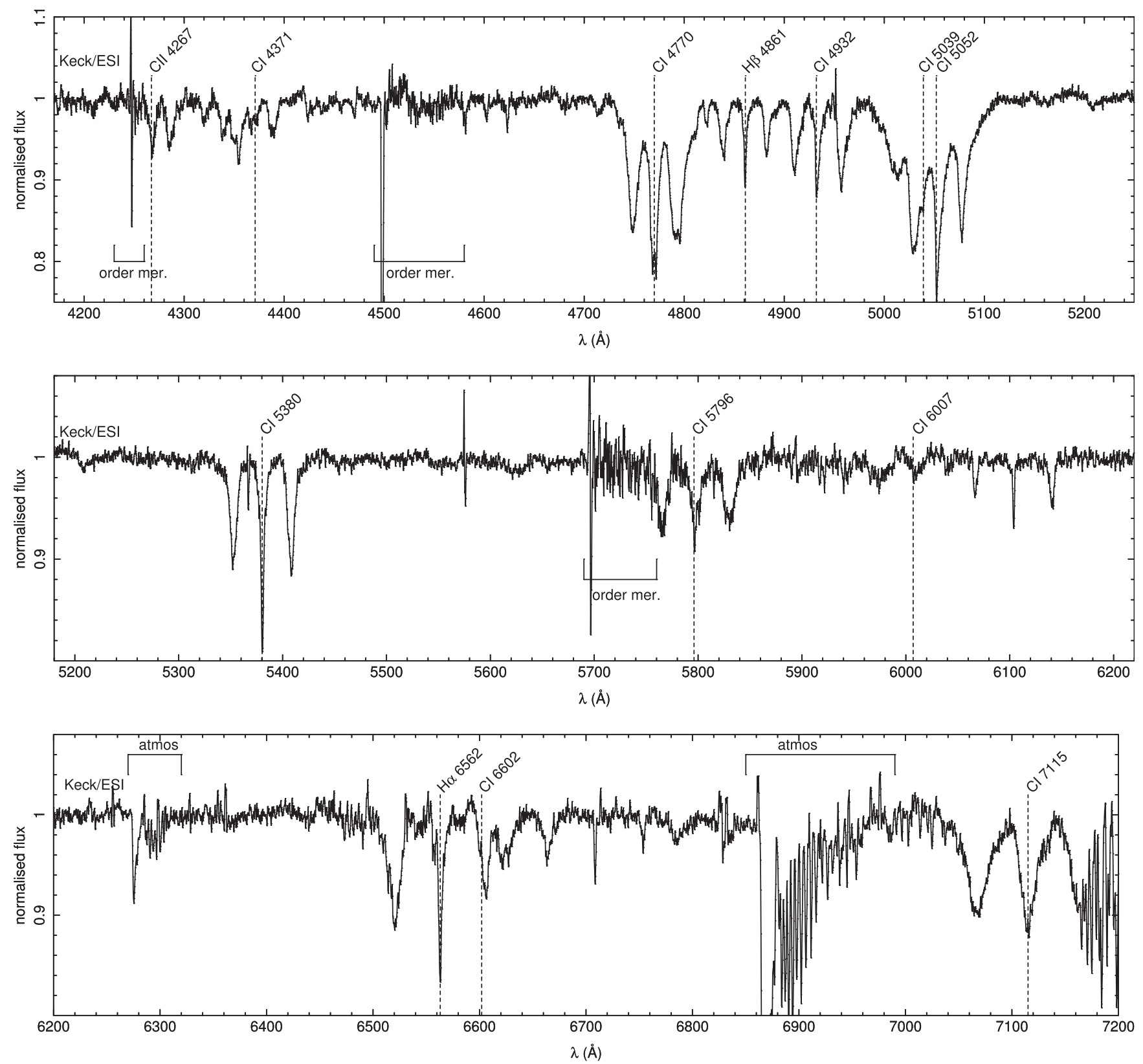

Figure 5. Average spectrum of OW J1753-3107, observed using the Echelle mode on the ESI instrument on the Keck telescope. The spectrum has been Gaussian-smoothed and is continuum- normalized. Spectral lines have been labelled. Regions of Echelle order mergers (order mer.) are indicated.

radial velocities are shown in Table 5, where the large error margins are the results of the low SNR in the single spectra, and the lack of strong unblended lines to measure. We were not able to detect any significant radial velocity variation in the spectral lines at the 35 -min period to an amplitude limit of $<5 \mathrm{~km} \mathrm{~s}^{-1}$, which suggests that OW J1753-3107 is not a UCB system.

\subsection{Spectral line fitting}

For spectral modelling, we obtained spectra of OW J1753-3107 using the Robert Stobie Spectrograph (RSS; Kobulnicky et al. 2003) on the 10-m Southern African Large Telescope (SALT; Buckley, Swart \& Meiring 2006). As shown in the spectroscopic observing $\log$ in Table 4, three 12-min exposures were made using the RSS in long-slit mode, with a 1.5 arcsec slit width. Furthermore, the grating pg1300 was used with a grating angle of 18.88 , providing a wavelength range of 3898-5981 ̊ with a wavelength sampling of $\sim 1.5$ pixels $\AA^{-1}$. The spectra were processed and extracted using standard IRAF (Tody 1986, 1993) and PYSALT (Crawford et al. 2010) routines, and LACOSMIC software (for cosmic ray reductions; van Dokkum 2001). A CuAr arc was used for wavelength calibration, and a spectrum of the standard star LTT 4364 was compared with for flux calibration.

The average combined SALT spectrum is shown in Fig. 6. To this spectrum, we fit an adaptation of the local thermodynamic equilibrium model atmospheres described in detail in Williams et al. (2013), which uses line splitting calculated in the Paschen-Back regime to form a grid of model atmospheres that consist of both helium and carbon. The model makes the assumptions that (a) the magnetic field does not affect the thermodynamic structure, (b) the 
Table 5. Radial velocity measurements for spectral lines of $\mathrm{H} \alpha, \mathrm{H} \beta$ and C I $\lambda 5380$ using FITSB2 (Napiwotzki et al. 2004) in the 18 KECK ESI spectra.

\begin{tabular}{ll}
\hline Heliocentric Julian Date & Velocity $\left(\mathrm{km} \mathrm{s}^{-1}\right)$ \\
\hline 2457540.9593649 & $70.5 \pm 6.7$ \\
2457540.9627950 & $68.0 \pm 5.5$ \\
2457540.9661938 & $77.0 \pm 6.3$ \\
2457540.9696060 & $66.4 \pm 6.4$ \\
2457540.9730071 & $70.8 \pm 8.3$ \\
2457540.9764268 & $79.8 \pm 8.6$ \\
2457540.9798291 & $82.6 \pm 7.0$ \\
2457540.9829443 & $82.7 \pm 9.3$ \\
2457540.9857036 & $84.3 \pm 15.8$ \\
2457540.9883721 & $73.5 \pm 8.2$ \\
2457541.0014171 & $68.3 \pm 23.6$ \\
2457541.0042447 & $91.1 \pm 8.9$ \\
2457541.0068692 & $83.0 \pm 8.7$ \\
2457541.0095776 & $70.7 \pm 9.3$ \\
2457541.0124886 & $72.2 \pm 14.1$ \\
2457541.0226497 & $87.3 \pm 16.0$ \\
2457541.0253813 & $81.4 \pm 12.3$ \\
2457541.0280880 & $75.3 \pm 11.4$ \\
\hline
\end{tabular}

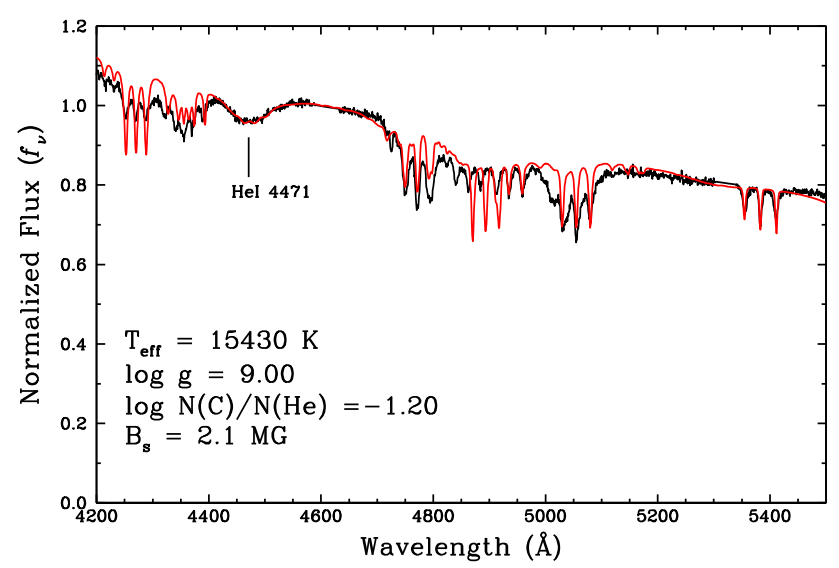

Figure 6. Average-combined and normalized SALT spectrum of OW J1753-3107 (black line), with the best-fitting model spectra overlain (red line). The physical properties, including the temperature $\left(T_{\text {eff }}\right)$, surface gravity $(g)$, magnetic field strength $\left(B_{z}\right)$ and carbon abundance $(N(\mathrm{C}) / N(\mathrm{He}))$ determined from the model fit are indicated. The model line strengths of the central $\mathrm{C}_{\mathrm{I}}$ lines around $4900 \AA, 4340 \AA$ and $\mathrm{He}_{\mathrm{I}} \lambda 4471$ are in agreement with the spectral data. However, the model does not fit several large-scale dips across the spectrum which are possibly due to spectral line identification issues.

target is undergoing normal radiative transfer and (c) $B_{z}$ is uniform over the surface of the star. See Williams et al. (2013) for a detailed description of this model.

Using a non-linear least-squares routine, a best-fitting solution is found for the spectra, as shown in Fig. 6. The solution is degenerate, and thus a change in one parameter $\left(T_{\text {eff }}, g\right.$ or $\left.N(\mathrm{C}) / N(\mathrm{He})\right)$ can be compensated for by a change in the other parameters. From visual inspection, the model manages to correctly replicate the line strengths of almost all the spectral features within this wavelength range. However, there appears to be large-scale discrepancies between the spectral data and the model fit (around the 4340 and $4900 \AA$ A regions), which is possibly due to spectral line identification issues. Furthermore, the model does not take into account any hydrogen in the atmosphere, and thus the possible $\mathrm{H} \beta$ absorption line (at $4861 \AA$ ) has not been fit. The values of $T_{\text {eff }}, g, B_{z}$ and

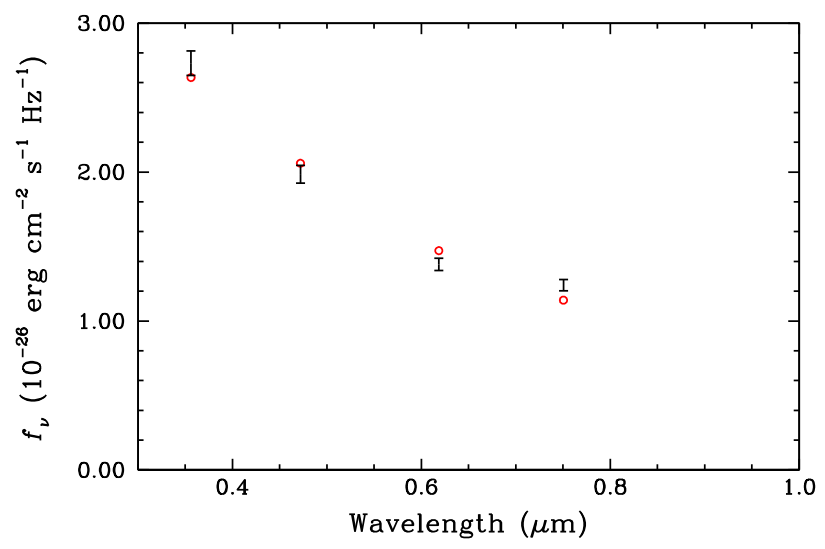

Figure 7. Comparison of the average fluxes (red circles) obtained from the best-fitting spectral model, and the fluxes from $u-, g_{-}, r$ - and $i$-band VPHAS+ colours (error bars) for the DQ OW J1753-3107. The best-fitting model is shown in Fig. 6.

$N(\mathrm{C}) / N(\mathrm{He})$, used to formulate the best-fitting model, are found as follows:

(i) A magnetic field strength of $B_{z}=2.1 \mathrm{MG}$ is determined by measuring the separation between the line components of the magnetically split C I triplet component at $5380 \AA$.

(ii) The broad He $\mathrm{I} \lambda 4471 \mathrm{dip}$ is used to calculate the carbon abundance, where $\log (N(\mathrm{C}) / N(\mathrm{He}))=-1.2$

(iii) The best-fitting model solution gives a temperature of $T_{\text {eff }}$ $=15430 \mathrm{~K}$, and a surface gravity of $\log (g)=9.0$. The $T_{\text {eff }}$ is determined from fitting the photometry [with $\log (g)$ fixed at 9.0 and $\log (N(\mathrm{C}) / N(\mathrm{He}))$ determined from the spectra].

Although the line strengths are generally well reproduced by the model, further modelling of improved spectra should provide more accurate estimates of the physical properties, which in turn can be used to find the mass of the target. In Fig. 7, we compare the VPHAS+ photometry obtained for OW J1753-3107 with photometry calculated from filter response curves folded to the best-fitting model spectra. This shows us that the model data agree with the VPHAS+ photometry to within $3 \sigma$ error bars.

\section{DISCUSSION}

The photometric and spectroscopic features of OW J1753-3107 reveal that it is a warm carbon-enriched DQ. Indeed, it can be considered a twin of the first warm DQ SDSS J1036+6522, but varying on a longer period and with a higher carbon abundance (see Table 6 for a comparison of the properties between these two warm DQs). In the following sections, we look at the similarities and differences between these two warm DQs, and discuss some key questions in hot and warm DQ research, namely (a) what is the nature of OW J1753-3107, and what does this imply for current theorized evolutionary channels, (b) what is the origin of the observed variations and (c) what does the magnetic nature of the system imply?

\subsection{Nature of the system}

The high abundance of carbon derived from the modelled atmospheres of hot DQs, along with such a constrained temperature range can be explained by the progenitor and evolutionary channel model proposed by Dufour et al. (2008). The carbon abundance observed 
Table 6. Comparison of the physical properties of the two warm DQs: OW J1753-3107 and SDSS J1036+6522. Properties include: the effective temperature $\left(T_{\text {eff }}\right)$, surface gravity $(\log (g))$, magnetic field strength $\left(B_{z}\right)$, carbon abundance $(\log (N(\mathrm{C}) / N(\mathrm{He})))$, period $(P)$ and amplitude $(A)$ of monoperiodic variations and the $g$-band magnitude (in Vega colour system).

\begin{tabular}{lrr}
\hline Property & OW J1753 -3107 & SDSS J1036+6522 \\
\hline$T_{\text {eff }}(\mathrm{K})$ & 15430 & 15500 \\
$\log (g)$ & 9.0 & 9.0 \\
$\log (N(\mathrm{C}) / N(\mathrm{He}))$ & -1.2 & -1.0 \\
$B_{z}(\mathrm{MG})$ & 2.1 & 3.0 \\
$P$ (min) & 35.5 & 18.6 \\
$A$ (per cent) & 1.3 & 0.4 \\
$g$ (mag) & 15.74 & 18.58 \\
\hline
\end{tabular}

in these sources are akin to those seen in massive PG1159 stars, such as H1054+65 (e.g. Werner et al. 2004) and RX J0439.8-6809 (Werner \& Rauch 2015). Both of these massive WDs are extremely hot $\left(T_{\text {eff }}>200000 \mathrm{~K}\right)$ and consist of atmospheres rich in carbon and oxygen. Their hydrogen- and helium-deficient atmospheres are believed to be the result of a very late and violent thermal pulse during the post-asymptotic-giant-branch phase which destroys most of the $\mathrm{H} / \mathrm{He}$ envelope. As the residual wind from this violent phase fades, gravitational settling causes the remaining helium, carbon and oxygen to separate, and the helium to form a thin shell surrounding the carbon-enriched mantle. The He atmosphere WD (with spectral type $\mathrm{DB}, T_{\mathrm{eff}} \approx 25000 \mathrm{~K}$ ) then cools down further and a convection zone in the carbon layer forms, causing the carbon to start to diffuse into the outer helium layer. The object is now a carbon-enriched hot DQ WD, with a temperature that is in the 18 000-24 $000 \mathrm{~K}$ range and a carbon abundance of $\log (N(\mathrm{C}) / N(\mathrm{He}))>1$. The hot DQ will then continue to cool as helium begins to resurface and, thus, it will transition via a 'warm' DQ phase into a second sequence of helium-enriched cool DQ WD.

The relatively low temperature and carbon abundance of OW J1753-3107 suggest it indeed resides in this transition state. Similarly to SDSS J1036+6522, the temperature of OW J1753-3107 ( $\left.T_{\text {eff }}=15430 \mathrm{~K}\right)$ is lower than the expected temperature range of hot DQs. In Table 7, we compare the colours (in the Vega magnitude system ${ }^{2}$ ) of OW J1753-3107 to other known warm or hot DQs, and show their location in colour-colour space in Fig. 8. First, we can see from this table that OW J1753-3107 is brighter by more than 2 mag in $u, g, r$ and $i$ colour bands. Secondly, the $u-g$ and $g-r$ colours of OW J1753-3107 appear consistent with other warm or hot DQs. This can also be seen in Fig. 8. Although the $r-i$ of our target appears relatively higher, this is possibly due to Galactic reddening as OW J1753-3107 is located towards the Galactic bulge. On the other hand, we deduce a photometric distance of $40.5_{-2.7}^{+3.4} \mathrm{pc}$ (assuming $\log (g)=9.0$ ), so the Galactic reddening may be small or negligible. We searched for evidence for proper motion using the SuperCOSMOS images (Hambly et al. 2001a). There is a clear offset in the position of OW 1753-3107 between the OW epoch (2014 July 29) and the SuperCOSMOS blue image (1974 June 17). The proper motion derived from SuperCOSMOS data for OW J1753-3107 is -0.7 mas

\footnotetext{
${ }^{2}$ Colours of the SDSS hot DQs have been transformed from the AB magnitude system into the Vega System using the equations of Blanton \& Roweis (2007). We note that there appears to be some uncertainty between the conversion from SDSS AB colours and VPHAS+ colours at the level of a few hundredths of mag in the $r$ band, rising to more than 1/10th of a mag in the $u$ band, see Drew et al. (2014) for details.
}

$\mathrm{yr}^{-1}$ in RA and -136 mas $\mathrm{yr}^{-1}$ in Dec. (Hambly et al. 2001b). The Gaia Data Release 2 scheduled for 2018 April will include the parallax and proper motion of OW J1753-3107 and will allow us to determine whether it is a member of the Galactic disc or halo.

From Fig. 8, it appears that the known sample of warm and hot DQs are located in a specific region of colour-colour space, implying that colour cuts can be applied to surveys such as OW in order to improve the future detection of new warm or hot DQs. However, with the current sample of these DQs, we are not yet able to confirm if warm and hot DQs cannot be differentiated based on their location in colour-colour space.

\subsection{The origin of variations}

One of the leading questions in hot DQ research concerns the origin of the observed variations. Early predictions by Montgomery et al. (2008) and Fontaine \& Brassard (2008) suggested that some hot DQs would be unstable to non-radial pulsations, which lead to the first discovery of variability in a hot DQ WD in SDSS J1425+5652 (Montgomery et al. 2008). The variations observed in the first five hot DQs, all with periods under $20 \mathrm{~min}$, were originally attributed to non-radial pulsations (Dufour et al. 2009, 2011), perhaps in analogy to large-amplitude rapidly oscillating A stars. However, the detection of a longer period variation of $2.1 \mathrm{~d}$ in the sixth discovered hot DQ SDSS J0005-1002 (with no evidence of a shorter period) was attributed to the rotation of a magnetic WD (Lawrie et al. 2013). Indeed, rapid rotation of the WD has now been proposed as the leading theory to explain the observed variations in hot DQs (Williams et al. 2016), supported by the apparent evidence that most hot DQs only exhibit single-mode variations (with related harmonics), as opposed to the multimode variations observed in pulsating WD. Although rotational periods are typically on the scale of hours to days, rotational periods as short as 10 s of minutes have been observed in some WDs (e.g. the 12-min rotations observed in the WD RE J0317-853; Barstow et al. 1995).

Since this rotation theory has been used to explain the 18.6-min variations seen in the warm DQ SDSS J1036+6522 (Williams et al. 2013), we can similarly use it as an explanation for the 35-min variations observed in OW J1753-3107. However, as both warm DQs are at a lower temperature than hot DQs and are thus theorized to be in a transition state, the source of the variations may be different to that of the other hot DQs (as suggested by Williams et al. 2013 for the variations observed in SDSS J1036+6522). Furthermore, unlike the short-period (under $20 \mathrm{~min}$ ) or $2.1 \mathrm{~d}$ variations of other hot DQs, OW J1753-3107 exhibits variations on a single period of $\sim 35 \mathrm{~min}$, with no evidence for other shorter or longer periods. Thus, it is likely the variations are not due to pulsations, but rather to the rotation of a magnetic WD.

\subsection{Magnetic behaviour}

Close to 70 per cent of hot DQs have been found to be strongly magnetic with magnetic field strengths $B_{z}>1 \mathrm{MG}$ (Dufour et al. 2011; Williams et al. 2016). The magnetic nature of some of the warm and hot DQs is also evident in the Zeeman splitting of their spectral lines (as in OW J1753-3107, see Fig. 1). By modelling these spectral features and measuring the degree of separation in the split carbon lines, Williams et al. (2013) were able to detect a magnetic field strength in SDSS J1036+6522 of $3.0 \pm 0.2$ MG. Similarly, the Zeeman-split carbon lines exhibited by OW J1753-3107 were used to calculate a magnetic field strength of $B_{z}=2.1 \mathrm{MG}$. 
Table 7. Colour properties of the eight warm and hot DQs. The colours of the previously known warm and hot DQ variables have been converted to lie on the Vega colour scale.

\begin{tabular}{lccccccr}
\hline Star ID & $\begin{array}{c}u \\
(\mathrm{mag})\end{array}$ & $\begin{array}{c}g \\
(\mathrm{mag})\end{array}$ & $\begin{array}{c}r \\
(\mathrm{mag})\end{array}$ & $\begin{array}{c}i \\
(\mathrm{mag})\end{array}$ & $\begin{array}{c}u-g \\
(\mathrm{mag})\end{array}$ & $\begin{array}{c}g-r \\
(\mathrm{mag})\end{array}$ & $\begin{array}{c}r-i \\
(\mathrm{mag})\end{array}$ \\
\hline Hot DQs & & & & & & & \\
SDSS J142625.70+575218.4 & 17.85 & 19.22 & 19.38 & 19.59 & -1.37 & -0.16 & -0.21 \\
SDSS J220029.08-074121.5 & 16.39 & 17.82 & 18.00 & 18.11 & -1.44 & -0.17 & -0.11 \\
SDSS J234843.30-094245.3 & 17.79 & 19.09 & 19.25 & 19.35 & -1.29 & -0.17 & -0.10 \\
SDSS J133710.19-002643.6 & 17.31 & 18.71 & 18.98 & 19.07 & -1.40 & -0.27 & -0.09 \\
SDSS J115305.54+005646.2 & 17.47 & 18.97 & 19.17 & 19.28 & -1.51 & -0.20 & -0.11 \\
SDSS J000555.90-100213.5 & 16.42 & 17.77 & 17.96 & 18.10 & -1.35 & -0.19 & -0.15 \\
Warm DQs & & & & & & & -0.10 \\
SDSS J103655.38+652252.0 & 17.39 & 18.58 & 18.68 & 18.78 & -1.19 & -0.10 & -0.10 \\
OW J175358.85-310728.9 & 14.40 & 15.74 & 15.89 & 15.80 & -1.34 & -0.16 & 0.09 \\
\hline
\end{tabular}

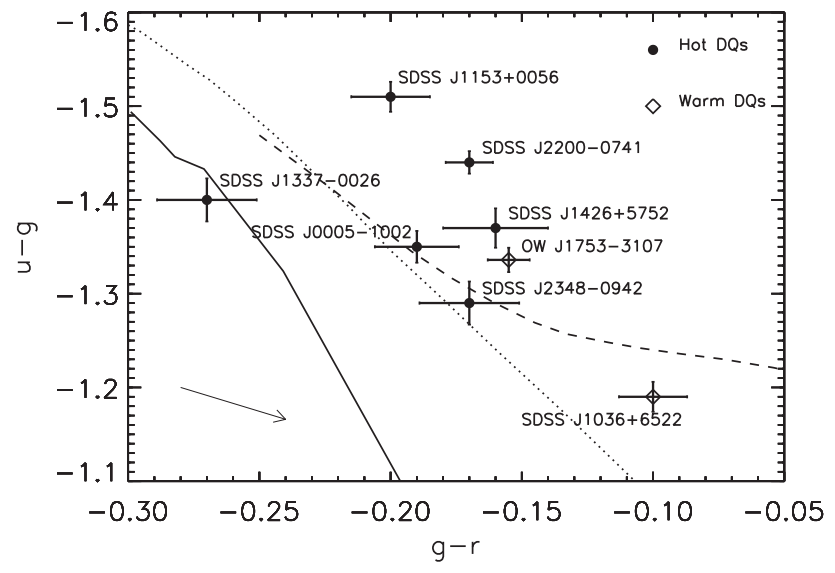

Figure 8. Distribution of all eight warm and hot DQs in $u-g$ versus $g-r$ space. We overlay the main sequence (MS) track (solid line), created using synthetic colours provided by Drew et al. (2014) for $R_{v}=3.1$ and extinction coefficient $\mathrm{A}_{0}=0$, indicated by 'Drew MS'. DA and DB tracks are shown as dotted and dashed lines, respectively, using synthetic colour-colour tracks with surface gravities of $\log (g)=8.0$ provided by Raddi et al. (2016). All colours are in the standard (Vega) system, and are listed in Table 7. All eight DQs, including our new discovery OW J1753-3107, are labelled. The reddening vector displayed in the bottom left of this figure represents $A_{V}=0.1$

It has been previously suggested that the observed non-linearities in the light-curve pulse shape of some hot DQs may be related to the presence of a strong magnetic field (e.g. Green et al. 2009). For example, the non-magnetic hot DQ SDSS J2348-0942 exhibits a sinusoidal pulse shape, whereas the hot DQ SDSS J2200-0741 exhibits non-sinusoidal variations (due to the presence of harmonics) and is strongly magnetic. However, SDSS J1036+6522 contradicts this theory, as it is strongly magnetic, yet exhibits sinusoidal pulse shapes (Williams et al. 2016). As the photometric analysis in this paper shows that OW J1753-3107 is also highly magnetic, and similarly shows a clear sinusoidal pulse shape, unchanging over $2 \mathrm{yr}$. Thus, this is further evidence against a non-linear pulse shape and magnetic field strength relationship.

This model of magneto-rotational variations can be addressed using high-resolution phase-resolved spectropolarimetry. We expect the degree of linear polarization to strongly change when the magnetic field poles rotate into/out of the line of sight.

\section{CONCLUSIONS}

In this paper, we present details of a new warm DQ OW J1753-3107, discovered in the OW Survey. Here, we summarize the main results of our photometric and spectroscopic analysis.

(i) OW J1753-3107 exhibits variations on a dominant period of 35.5452 (2) min, with no clear evidence for harmonics or other non-related periods. A single-sinusoidal model provided a good fit for all follow-up light curves. Furthermore, the 35-min period has remained constant over a 2-yr span from 2014 to 2016.

(ii) The follow-up spectra of OW J1753-3107 reveal strong evidence for $\mathrm{C}_{\mathrm{I}}$ and $\mathrm{C}_{\mathrm{II}}$ lines in absorption, along with a broad $\mathrm{He}_{\mathrm{I}}$ $\lambda 4471$ dip and possible hydrogen absorption lines. With the exception of the hydrogen features, it is very similar in appearance to the spectrum of the warm DQ SDSS J1036+6522.

(iii) OW J1753-3107 has a temperature of $T_{\text {eff }}=15430 \mathrm{~K}$, and a carbon abundance of $\log (N(\mathrm{C}) / N(\mathrm{He}))=-1.2$. As in the case of SDSS J1036+6522, these values are lower than those of known hot DQs. Thus, OW J1753-3107 can be considered to be a warm DQ, believed to exist in a transition state between the hot carbonenriched DQ and the cooler helium-dominant DQ.

(iv) The current sample of known warm and hot DQs appear to reside in a distinct region of colour-colour space. However, it is currently not possible to differentiate between hot DQs and warm DQs using their location in this plane.

(v) The mean magnetic field strength of OWJ1753-3107 is $B_{z}=2.1 \mathrm{MG}$, slightly lower than that of SDSS J1036+6522. The sinusoidal pulse shape of OW J1753-3107 provides further evidence to suggest that there is no relationship between non-linearities in the pulse shape of warm or hot DQs and their magnetic field strength.

(vi) The origin of the 35-min variation seen in OW J1753-3107 is currently believed to be due to the rotation of the WD. A pulsational model has been ruled out since there is no evidence for multimode variations, and the period is longer than expected from the pulsational periods observed in other WDs. Furthermore, there is no evidence of the emission lines in its spectrum that would indicate it is an interacting binary star system undergoing accretion. The lack of significant radial velocity variations at the 35-min period, to an amplitude limit of $<5 \mathrm{~km} \mathrm{~s}^{-1}$, appears to rule out that $\mathrm{OW}$ J1753-3107 is a UCB system.

(vii) Dedicated surveys are needed in order to increase and characterize the known population of the hot and warm DQs. This will help to answer the most pressing questions in this research field, 
such as the origin of the variations and the type of evolutionary channels that the hot and warm DQs may follow.

\section{ACKNOWLEDGEMENTS}

The authors gratefully acknowledge funding from the Erasmus Mundus Programme SAPIENT, the National Research Foundation of South Africa (NRF), the Nederlandse Organisatie voor Wetenschappelijk Onderzoek (the Dutch Organisation for Science Research), Radboud University and the University of Cape Town. PD acknowledges support from NSERC (Canada). Armagh Observatory is core funded by the Northern Ireland Executive. The ESO observations used in this paper are based on observations made with ESO Telescopes at the La Silla Paranal Observatory under programme ID 093.D-0753(A) as part of the Dutch GTO time on OmegaCAM and 177.D-3023 (VPHAS+). This paper uses observations made at the South African Astronomical Observatory (SAAO). Some of the observations reported in this paper were obtained with the Southern African Large Telescope (SALT) under the programme 2016-1-SCI-015 (PI: Sally Macfarlane). This research is supported by the NWO/NRF Bilateral agreement supporting astronomical research. We thank the anonymous referee for the useful comments that have helped to improve the paper.

\section{REFERENCES}

Alam S. et al., 2015, ApJS, 219, 12

Althaus L. G., Córsico A. H., Isern J., García-Berro E., 2010, A\&AR, 18, 471

Barlow B. N., Dunlap B. H., Rosen R., Clemens J. C., 2008, ApJ, 688, L95

Barstow M. A., Jordan S., O’Donoghue D., Burleigh M. R., Napiwotzki R., Harrop-Allin M. K., 1995, MNRAS, 277, 971

Blanton M. R., Roweis S., 2007, AJ, 133, 734

Buckley D. A. H., Swart G. P., Meiring J. G., 2006, in Stepp L. M., ed., Proc. SPIE Conf. Ser. Vol. 6267, Ground-based and Airborne Telescopes. SPIE, Bellingham, p. $62670 \mathrm{Z}$

Capaccioli M., Schipani P., 2011, The Messenger, 146, 2

Coppejans R. et al., 2013, PASP, 125, 976

Crawford S. M. et al., 2010, in Silva D. R., Peck A. B., Soifer T., eds, Proc. SPIE Conf. Ser. Vol. 7737, Observatory Operations: Strategies, Processes, and Systems III. SPIE, Bellingham, p. 773725

Dolez N. et al., 2006, A\&A, 446, 237

Drew J. E. et al., 2014, MNRAS, 440, 2036

Dufour P., Bergeron P., Fontaine G., 2005, ApJ, 627, 404

Dufour P., Liebert J., Fontaine G., Behara N., 2007, Nature, 450, 522

Dufour P., Fontaine G., Liebert J., Schmidt G. D., Behara N., 2008, ApJ, 683, 978

Dufour P., Green E. M., Fontaine G., Brassard P., Francoeur M., Latour M., 2009, ApJ, 703, 240

Dufour P., Béland S., Fontaine G., Chayer P., Bergeron P., 2011, ApJ, 733, L19

Dufour P., Vornanen T., Bergeron P., Fontaine A. B., 2013, in Krzesinsky J., Stachowski G., Moskalik P., Bajan K., eds, ASP Conf. Proc. Vol. 469, 18th European White Dwarf Workshop. Astron. Soc. Pac., San Francisco, p. 167
Dunlap B. H., Barlow B. N., Clemens J. C., 2010, ApJ, 720, L159

Fontaine G., Brassard P., 2008, PASP, 120, 1043

Fortier A., Dufour P., 2015, in Dufour P., Bergeron P., Fontaine G., eds, ASP Conf. Ser. Vol. 493, 19th European Workshop on White Dwarfs. Astron. Soc. Pac., San Francisco, p. 41

Green E. M., Dufour P., Fontaine G., Brassard P., 2009, ApJ, 702, 1593

Hambly N. C. et al., 2001a, MNRAS, 326, 1279

Hambly N. C., Davenhall A. C., Irwin M. J., MacGillivray H. T., 2001b, MNRAS, 326, 1315

Hartman J. D., Gaudi B. S., Holman M. J., McLeod B. A., Stanek K. Z., Barranco J. A., Pinsonneault M. H., Kalirai J. S., 2008, ApJ, 675, 1254

Henden A. A., Levine S. E., Terrell D., Smith T. C., Welch D., 2012, J. Am. Assoc. Var. Star Obs., 40, 430

Hermes J. J. et al., 2012, ApJ, 757, L21

Kobulnicky H. A., Nordsieck K. H., Burgh E. B., Smith M. P., Percival J. W., Williams T. B., O’Donoghue D., 2003, in Iye M., Moorwood A. F. M., eds, Proc. SPIE Conf. Ser. Vol. 4841, Instrument Design and Performance for Optical/Infrared Ground-based Telescopes. SPIE, Bellingham, p. 1634

Kuijken K. et al., 2002, The Messenger, 110, 15

Landolt A. U., 1968, ApJ, 153, 151

Lawrie K. A., Burleigh M. R., Dufour P., Hodgkin S. T., 2013, MNRAS, 433,1599

Liebert J. et al., 2003, AJ, 126, 2521

Macfarlane S. A., Toma R., Ramsay G., Groot P. J., Woudt P. A., Drew J. E., Barentsen G., Eislöffel J., 2015, MNRAS, 454, 507

Montgomery M. H., Williams K. A., Winget D. E., Dufour P., DeGennaro S., Liebert J., 2008, ApJ, 678, L51

Napiwotzki R. et al., 2004, in Hilditch R. W., Hensberge H., Pavlovski K., eds, ASP Conf. Ser. Vol. 318, Spectroscopically and Spatially Resolving the Components of the Close Binary Stars. Astron. Soc. Pac., San Francisco, p. 402

Raddi R. et al., 2016, MNRAS, 457, 1988

Sheinis A. I., Bolte M., Epps H. W., Kibrick R. I., Miller J. S., Radovan M. V., Bigelow B. C., Sutin B. M., 2002, PASP, 114, 851

Tody D., 1986, in Crawford D. L., ed., Proc. SPIE Conf. Ser. Vol. 627, Instrumentation in Astronomy VI. SPIE, Bellingham, p. 733

Tody D., 1993, in Hanisch R. J., Brissenden R. J. V., Barnes J., eds, ASP Conf. Ser. Vol. 52, Astronomical Data Analysis Software and Systems II. Astron. Soc. Pac., San Francisco, p. 173

Toma R. et al., 2016, MNRAS, 463, 1099

van Dokkum P. G., 2001, PASP, 113, 1420

Werner K., Rauch T., 2015, A\&A, 584, A19

Werner K., Rauch T., Napiwotzki R., Christlieb N., Reimers D., Karl C. A., 2004, A\&A, 424, 657

Williams K. A., Liebert J., Bolte M., Hanson R. B., 2006, ApJ, 643, L127

Williams K. A. et al., 2013, ApJ, 769, 123

Williams K. A., Montgomery M. H., Winget D. E., Falcon R. E., Bierwagen M., 2016, ApJ, 817, 27

Winget D. E., Kepler S. O., 2008, ARA\&A, 46, 157

This paper has been typeset from a $\mathrm{T}_{\mathrm{E}} \mathrm{X} / \mathrm{LAT} \mathrm{E}$ file prepared by the author. 